\section{GP26 TRANSCATHETER CLOSURE OF SECUNDUM ATRIAL SEPTAL DEFECT: FOLLOW-UP RESULTS FROM A TERTIARY CENTER}

${ }^{1}$ Burcu Kilinç*, ${ }^{2}$ Irfan Levent Saltik. 'Department of Pediatrics, Istanbul UniversityCerrahpaşa, Cerrahpaşa Medical Faculty, istanbul, Turkey; ${ }^{2}$ Department of Pediatric Cardiology, Istanbul University-Cerrahpaşa, Cerrahpaşa Medical Faculty, istanbul, Turkey

\subsection{6/archdischild-2019-epa.93}

Background and objectives Atrial septal defect (ASD) is a deficiency in the atrial septum leading to an abnormal communication between the right and left atria. Defects of the atrial septum are the third most common type of congenital heart disease comprising $6 \%$ of all lesions. Transcatheter closure of secundum type ASD is an alternative to surgical closure in many cases when conditions are appropriate. Studies have shown that closure of secundum type atrial septal defects with devices is a safe and successful method. In this study, we planned to share the demographic datas and postoperative follow-up results of patients with secundum atrial septal defect undergoing transcatheter closure.

Methods Data of patients underwent transcatheter closure of secundum type atrial septal defect between 2004 to 2017 was investigated retrospectively. Gender, age at intervention, defect size (with transthoracic, transesophageal echocardiography), procedure duration, fluoroscopy time, periprocedural complications, residual shunt existence and long term follow-up results were collected.

Results In 179 patients (41\% males;\%10 adults, median age, 8,1 years [1,3 to 58,6]; weight, $28 \mathrm{~kg}$ [11 to 90]), admitted to catheterization for ASD closure. Median ASD size was 13 $\mathrm{mm}$ (6 to 30$) ; 74(41 \%)$ patients had a large ASD $(\geq 12$ $\mathrm{mm})$. Suitable defects for closure were 165 of 179 patients. Three types of devices were used during procedure most used being Amplatzer Septal Occluder. Procedural success rate was $95,7 \%$. No death was observed but periprocedural complications occurred in 3 patients (1,6\%). Periprocedural complications were AV(atrioventricular) block, atrial flutter and brachial plexus paralysis. After a median follow-up of 2,8 years (range 6 months to 13,6 years; 16 patients [10\%] followed $>10$ years), delayed major complications such as death, cardiac erosion, infective endocarditis were not experienced. Delayed minor complications were supraventricular extrasystole not required treatment and mild mitral regurgitation worsening in 2 patients (1,3\%). The rate of residual shunt was $1,3 \%$ at one year follow-up and all shunts were mild.

Conclusions Transcatheter ASD closure is safe in children with a minimal rate of periprocedural complications and a favorable long-term outcome, especially with no death or major complications. Residual shunt ratio is also low and insignificant. Transcatheter ASD closure can be done safely in experienced centers as an alternative treatment to surgery.

\section{GP27 EXPERIENCE OF USING PULSE OXIMETRY SCREENING IN NEWBORNS TO IDENTIFY PULMONARY HYPERTENSION AND CRITICAL CONGENITAL HEART DEFECTS IN RUSSIA}

Dinara Sadykova, Dina Sabirova, Anna Babintseva, Natalya Kustova*. Kazan State Medical University, Kazan, Russian Federation

10.1136/archdischild-2019-epa.94
Background The urgency of the problem is determined by the high infant mortality and disability associated with critical CHD (CCHD) and persistent pulmonary hypertension in newborns (PPHN). The difficulty of diagnosing critical CHD and persistent pulmonary hypertension in newborns leads to a delayed accurate diagnosis, which is the cause of late hospitalization in specialized hospitals in a difficult and often critical condition. This research paper presents a method for the early detection of newborns with PPHN and critical CHD using pulse oximetry, which differs from the existing methods by the beginning of the saturation measurement time, as well as an extended diagnostic search for conditions that threaten the life of the newborn.

Purpose Assess the diagnostic value of pulse oximetry conducted by an infant at an earlier period (at 3 o'clock after birth) in order to identify newborns with persistent pulmonary hypertension and critical congenital heart defects.

Methods The work was carried out on the basis of the maternity hospital of the Republic of Tatarstan of the Russian Federation. The study included all newborns born alive for a period of more than 34 weeks of gestational age without prenatally confirmed pathology of the cardiovascular and pulmonary systems. Newborns are screened twice: in the third hour of life, which ensures early diagnosis of persistent pulmonary hypertension of newborns and critical CHD, and on the 3rd day after birth, that is, before vaccination against tuberculosis, since vaccination may worsen the condition of a newborn with undiagnosed pathology. Pulse oximetry is performed at any time if the newborn has any clinical manifestations of critical conditions.

Results For the period from April 2016 to December 2017 screening covered 19,110 newborns. Positive screening results were obtained in 242 patients. Thanks to screening, in 29 newborns in the first hours of life, congenital heart defects that were not diagnosed prenatal were detected, 6 of them were critical. All newborns with critical CHD were transferred for emergency indications to the cardiac surgery department and successfully operated. Persistent pulmonary hypertension was detected in 34 patients. Congenital pneumonia was diagnosed in 104 newborns.

Conclusion Early detection of life-threatening conditions allowed time to begin therapy, to avoid deaths and critical complications. In addition, during the screening, other concomitant conditions accompanied by hypoxemia were also diagnosed. In addition to the early detection of critical CHD using pulse oximetric screening, other diseases can be identified, including persistent pulmonary hypertension of the newborn and congenital pneumonia.

\section{GP29 THE EFFECT OF 2015 REVISED JONES CRITERIA ON ACUTE RHEUMATIC FEVER DIAGNOSIS}

${ }^{1}$ Tugcem Keskin, ${ }^{1}$ Pelin Kosger, ${ }^{1}$ Hikmet Kiztanir, ${ }^{2}$ Birgul Kirel, ${ }^{1}$ Birsen Ucar*. ${ }^{1}$ Eskisehir Osmangazi University Faculty of Medicine, Department of Pediatric Cardiology, Eskisehir, Turkey; ${ }^{2}$ Eskisehir Osmangazi University Faculty of Medicine, Department of Pediatric Endocrinology, Eskisehir, Turkey

\subsection{6/archdischild-2019-epa.95}

Objective Consistent with the high risk countries, the incidence of acute rheumatic fever (ARF) in school age children is reported to be $21-42 / 100.000$ in Turkey. According to the Jones criteria, which the American Heart Association updated in 2015, monoarthritis and polyarthritis were major, fever 